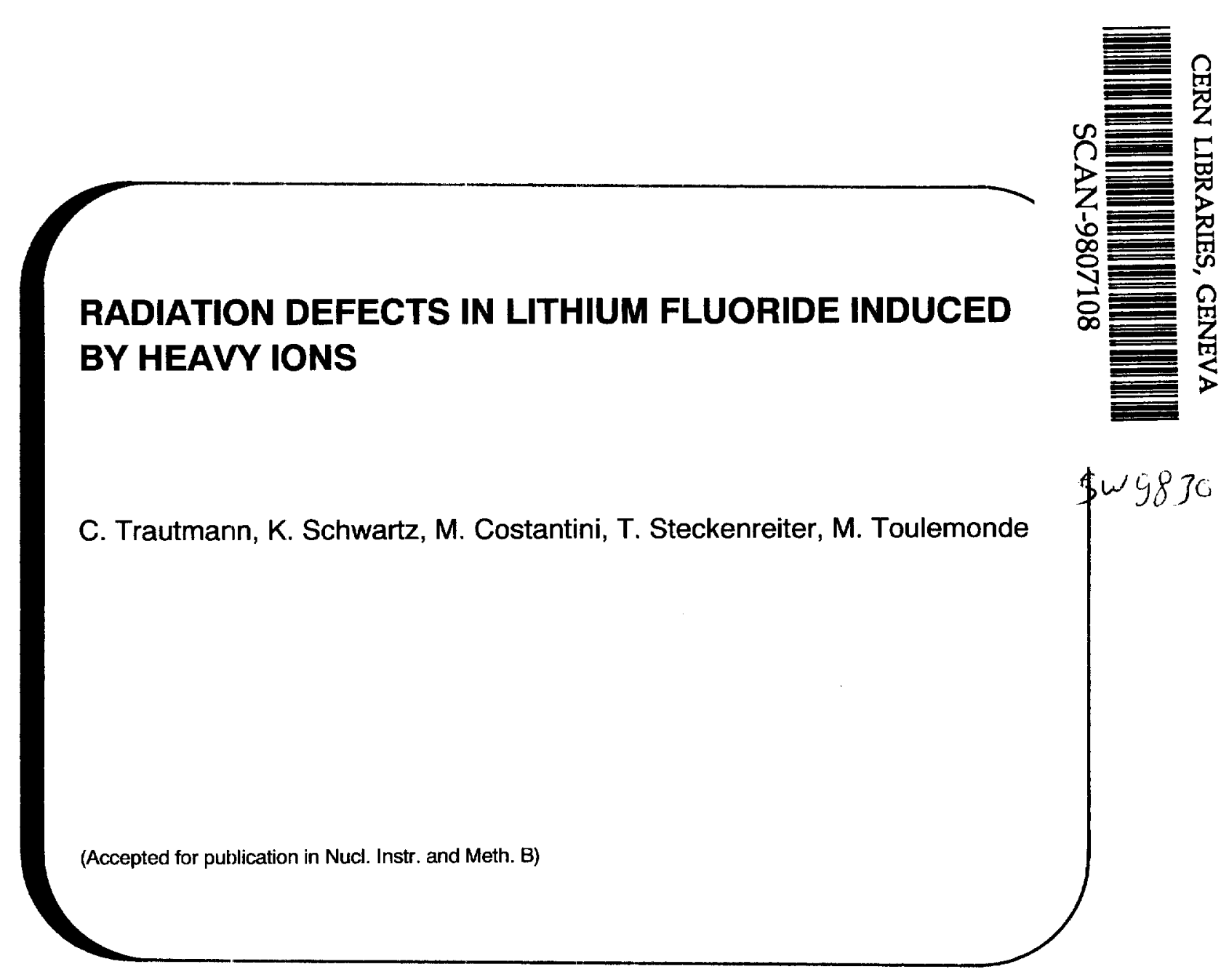

Gesellschaft für Schwerionenforschung $\mathrm{mbH}$

Planckstraße 1 - D-64291 Darmstadt • Germany Postfach 110552 • D-64220 Darmstadt - Germany 


\title{
Radiation defects in lithium fluoride induced by heavy ions
}

\author{
C. Trautmann ${ }^{*}$, K. Schwartz ${ }^{1}$, J.M. Costantini ${ }^{2}$, T. Steckenreiter ${ }^{1}$, and M. Toulemonde ${ }^{3}$ \\ 1 Gesellschaft für Schwerionenforschung, Planckstr. 1, 64291 Darmstadt, Germany \\ 2 CEA/DPTA/SPMC, BP 12, 91680 Bruyères-Le-Châtel, France \\ 3 CIRIL, Laboratoire commun CEA/CNRS, BP 5133, 14070 Caen-cedex 5, France \\ * Corresponding author: e-mail: C.Trautmann@gsi.de \\ Tel.: $\quad-49-6159-712716$ \\ Fax.: $\quad-49-6159-712179$
}

PACS numbers: 61.10Eq, 61.72.Cc, 61.72.Dd, 61.72.Ji, 61.72Ff, 61.72.Cc, 61.80.Jh, 71.35Ee

\begin{abstract}
Single crystals of lithium fluoride were irradiated with various species of heavy ions in the energy regime between 1 and $30 \mathrm{MeV} / \mathrm{u}$. The induced radiation damage was studied with techniques such as optical absorption spectroscopy, small-angle $\mathrm{x}$-ray scattering, chemical etching and profilometry, complemented by annealing experiments. Clear evidence is given for a complex track structure and defect morphology. Single defects such as $F$-centers are produced in a large halo of several tens of nanometers around the ion trajectory. The defect creation in this zone is similar to that under conventional radiation. For heavy ions above a critical energy loss of $10 \mathrm{keV} / \mathrm{nm}$, new effects occur within a very small core region of 2-4 $\mathrm{nm}$ in diameter. The damage in this zone is responsible for chemical etching and for a characteristic anisotropic $\mathrm{x}$-ray scattering. It is assumed that in this core, complex defect aggregates (e.g., cluster of color centers, molecular anions and vacancies) are created. Their formation is only slightly influenced by the irradiation temperature and takes place even at $15 \mathrm{~K}$ where diffusion processes of primary defects are frozen. Furthermore, irradiation with heavy ions leads to pronounced swelling effects which can be related to an intermediate zone of around $10 \mathrm{~nm}$ around the ion path.
\end{abstract}




\section{Introduction}

The formation of ion tracks in alkali halides has attracted our attention because, many details are known about the defect creation under various types of radiation (photons, electrons, neutrons, and ions). Without doubt, the problem has owed its fascination to the simplicity of the ionic lattice of such crystals and the ease with which the structure can be damaged. Under ionising radiation, the damage is mainly created in the anion sublattice. The primary defects are created via self-trapping of excitons and the subsequent nonradiative decay. At room temperature, the most significant type of defects are $F$-centers (an electron on an anion vacancy), $F_{2}$-centers, and the complementary hole centers (Fig. 1) [1-4].

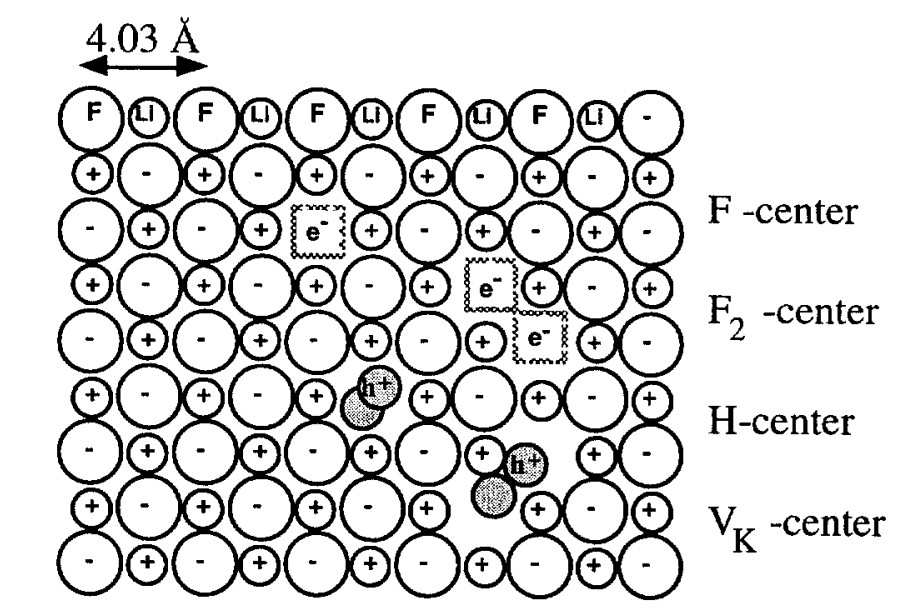

Fig. 1 Primary defects in lithium fluoride

Depending on the material, such point defects subsist up to a very high level of energy deposition which allows the study of special effects, for instance the deviation from linearity in the defect production, saturation effects, and the formation of complex aggregates (e.g., cluster of color centers, molecular anions and vacancies $[5,6])$. Under high dose irradiation with neutrons or $\mathrm{x}$-rays, even metallic colloids can be created if a critical defect concentration is reached and if the aggregation process is initiated by thermal or radiation enhanced diffusion [7-10].

Among all ionic crystals, LiF has the strongest ionic binding and the largest band gap $(14 \mathrm{eV})$. For a long time, $\mathrm{LiF}$ has successfully been used as radiation dosimeter of high 
sensitivity that can be applied even up to a very high dose of about $100 \mathrm{MGy}$ [11]. Due to its high transmission in the wavelength regime between $100 \mathrm{~nm}$ and a few micrometers, these crystals find a wide field of application as special optical windows. Furthermore, it should be mentioned that $\mathrm{LiF}$ is also attractive as laser material with tuneable color centers [12].

The aim of the here, described investigations was to gain a better understanding concerning the specificity of the damage produced when irradiated LiF with swift heavy ions. At energies above about $1 \mathrm{MeV} / \mathrm{u}$, elastic collisions between the projectile ion and the target atoms can be neglected, and the interaction is characterised by almost pure electronic excitation of the target atoms. The primary ionisation and excitation processes and the following electron cascades occur within a very short time of $10^{-17}$ to $10^{-14} \mathrm{~s}$, which is much shorter than the time necessary to create defects via lattice relaxation $\left(10^{-12}-10^{-11} \mathrm{~s}\right)$ [13]. Another significant difference to low ionising radiation (e.g., electrons, gammas or $\mathrm{x}$-rays) is the inhomogeneous distribution of the absorbed dose. It originates from the fact that each individual ion deposits its energy with an approximately $1 / \mathrm{r}^{2}$ dependence on the radial distance from the trajectory. Such a dose gradient could have a strong influence on the defect creation mechanisms and on the nature of the damage. In particular close to the ion path, extremely high local doses are reached (MGy within a radius of a nanometer), difficult to obtain by any other energy deposition process.

While special ion-induced effects have been studied in a wide range of insulating materials (e.g. polymers $[14,15]$ and oxides $[16,17])$, only a few investigations have been made in ionirradiated alkali halides. In most cases, the creation of single defects was studied by means of optical spectroscopy using lighter ions of rather low stopping powers [18-21]. Special techniques analysing more complex defects such as aggregates of $F$-centers or colloids were not applied.

In this paper, we are presenting the results of new experiments on ion irradiated lithium fluoride obtained by combining various techniques such as optical absorption spectroscopy, chemical etching, small-angle x-ray scattering (SAXS) and profilometry, complemented by 
annealing experiments. The resulting complex information provide us with a much better understanding of the damage morphology and structure of tracks in particular of heavy ions.

\section{Irradiation}

Thin platelets with a typical thickness of a few hundred micrometers were cleaved from a single-crystal block of high purity lithium fluoride. The samples were irradiated under normal incidence with $\mathrm{Cu}(60 \mathrm{MeV})$ and $\mathrm{S}(50 \mathrm{MeV})$ ions at the 7 Megavolt Van de Graaff tandem (Bruyères-le-Châtel), with C (11.1 MeV/u), Mo (4.4 MeV/u), and Pb (4 and $29 \mathrm{MeV} / \mathrm{u}$ ) ions at the GANIL accelerator (Caen), and with various ion species between $\mathrm{Ni}$ and $\mathrm{U}$ ions (11.4 $\mathrm{MeV} / \mathrm{u}$ ) at the UNILAC of the GSI (Darmstadt). During some irradiations, thin aluminium or polymer foils were placed in front of the crystals in order to vary the energy and energy loss of the ions when impinging on the sample surface. The projected ion range varied from about $10 \mu \mathrm{m}$ (for $\mathrm{Cu}$ and $\mathrm{S}$ ions), to $100 \mu \mathrm{m}$ (for $\mathrm{Ni}$ up to $\mathrm{U}$ ions of $11.4 \mathrm{MeV} / \mathrm{u}$ ), and to more than $200 \mu \mathrm{m}$ (for $\mathrm{C}(11 \mathrm{MeV} / \mathrm{u})$ and $\mathrm{Pb}(29 \mathrm{MeV} / \mathrm{u})$ ions). The range was in most cases less than the thickness of the crystals. The flux of the ion beam was of the order of $10^{9}$ ions $\mathrm{s}^{-1} \mathrm{~cm}^{-2}$ at the tandem Van de Graaff accelerator and between 2 and $4 \times 10^{8}$ ions s $\mathrm{s}^{-1} \mathrm{~cm}^{-2}$ at the GANIL and GSI accelerators.

\section{Optical absorption spectroscopy}

The irradiation of the LiF crystals with heavy ions lead to a strong coloration between yellow and dark brown depending on the fluence and range of the ions. This effect is due to the creation of $F$-centers, aggregates of $F$-centers, or even small colloids formed in the irradiated volume. They were studied in the spectral range between 200 and $900 \mathrm{~nm}$ using a UV-visible double-beam spectrometer. As quoted in the literature, the absorption band of $F$ and $F_{2}$-centers was found at a wavelength of $245 \mathrm{~nm}$ and $445 \mathrm{~nm}$, respectively. From the Smakula-Dexter formula we determined the concentration per $\mathrm{cm}^{-2}$ of the $F$-centers $\left(\mathrm{n}_{\mathrm{F}}=\right.$ $\left.9.48 \times 10^{15} \times \mathrm{A}\right)$ and of the $F_{2}$-centers $\left(\mathrm{n}_{\mathrm{F}_{2}}=4.42 \times 10^{15} \times \mathrm{A}\right)$ at the maximum $\mathrm{A}$ of their absorbance band $[18,22]$. For the corresponding volume concentration $N_{F}$ and $N_{F 2}$, the 
thickness of the irradiated volume has to be taken into account $\left(N_{F}=n_{F} / R\right.$ and $N_{F_{2}}=n_{F_{2}} / R$, where $\mathrm{R}$ is the ion range).

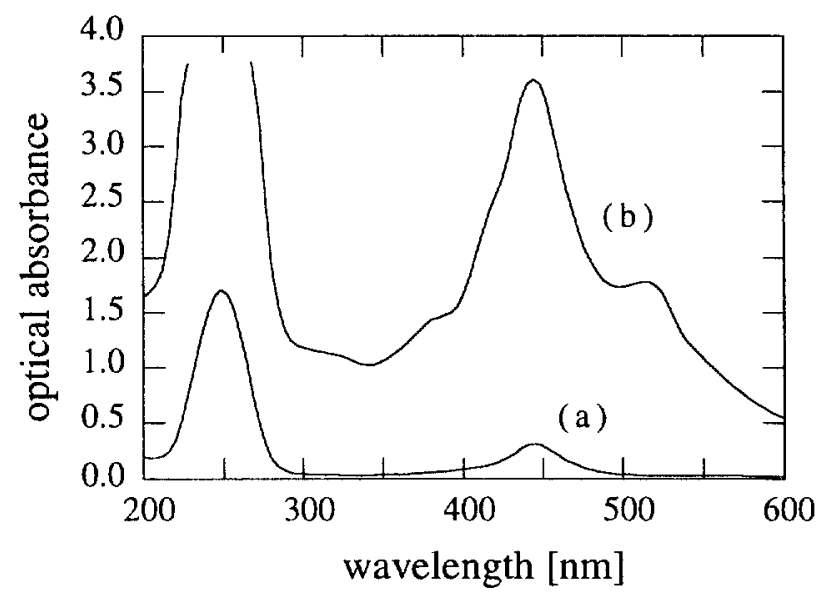

Fig. 2 Absorption spectra of LiF crystals irradiated with $\mathrm{Bi}$ ions $(10.9 \mathrm{MeV} / \mathrm{u})$ at a fluence of (a) $10^{10} \mathrm{~cm}^{-2}$ and (b) $2 \times 10^{11} \mathrm{~cm}^{-2}$.

Figure 2 shows a typical absorption spectrum of a crystal irradiated with Bi ions (10.9 $\mathrm{MeV} / \mathrm{u}$ ) of a rather low fluence (curve a). At higher fluences (curve b), the spectrum is more complex exhibiting peak shoulders at around $315,375,518$, and $540 \mathrm{~nm}$. These new bands can be assigned to color center aggregates such as $F_{3}$-centers $(320,380 \mathrm{~nm}), F_{4}$-centers $(518$, $540 \mathrm{~nm}$ ), and possibly to Li colloids [23]. It should be mentioned that for colloids in LiF the dependence of the absorption band on shape and size is not well known. Various absorption bands are expected in the ultraviolet and visible spectral range [5,7,24], and consequently might overlap with the bands of other color centers. For this reason, we did not deconvolute such complex spectra but focused mainly on the creation of single color centers. 


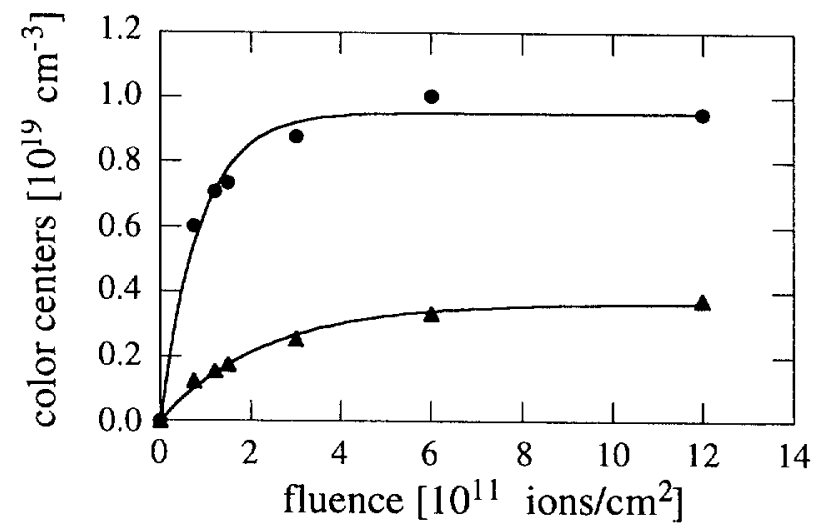

Fig. 3 Concentration of $F$-centers (circles) and $F_{2}$-centers (triangles) as a function of the fluence of $\mathrm{Pb}$ ions with an energy of $4.0 \mathrm{MeV} / \mathrm{u}$.

Figure 3 shows the evolution of the number of $F$-and $F_{2}$-centers under the irradiation with $\mathrm{Pb}$ ions of $4 \mathrm{MeV} / \mathrm{u}$. At sufficiently high fluences, the concentration of both color centers reach saturation. This observation is ascribed to overlapping effects of individual ion tracks. From such a saturation curve, the radius of the track can be deduced in which e.g. mainly $F$ centers are created. According to the model proposed by Thévenard et al. [20], the situation is simplified by supposing that the $F$-centers are homogeneously distributed in a cylindrical volume around the ion trajectory. Whenever two neighbouring tracks overlap, $F$-centers are assumed to aggregate and the creation of additional single $F$-centers is neglected. Depending on the radial extension of the track, the concentration of $F$-centers as a function of the fluence will reach saturation, when the entire crystal surface is covered by tracks. The mathematical description of this evolution is given by

$$
n_{F}=n_{S}\left(1-\exp \left(-\pi r_{F}^{2} \times \Phi\right)\right)
$$

where $\mathrm{n}_{\mathrm{F}}\left[\mathrm{cm}^{-2}\right]$ is the experimentally observed number of $F$-centers per unit of the sample surface irradiated with an ion fluence $\Phi\left[i o n s / \mathrm{cm}^{2}\right], \mathrm{n}_{\mathrm{S}}\left[\mathrm{cm}^{-2}\right]$ is the saturation density of $F$ centers, and $\mathrm{r}_{\mathrm{F}}$ is the radius in which single $F$-centers dominate. The curve in Fig. 3 results from a fit of the experimental $F$-center data by means of Eq. 1. For $\mathrm{Pb}$ ions, the corresponding track radius $\mathrm{r}_{\mathrm{F}}$ is about $20 \mathrm{~nm}$.

Another interesting parameter deduced from the fit of the $F$-center curve in Fig. 3 is the 
saturation level of about $10^{19} \mathrm{~cm}^{-3}$. For LiF and other alkali halides under gamma or electron irradiation in the high dose regime, such a concentration is typical for the onset of aggregation processes $[7,23,25]$.

In order to compare the defect creation of various ion species, we analysed the $F$-center concentration as a function of the total absorbed dose which depends on the particle fluence, the mass density $\left(2.635 \mathrm{~g} / \mathrm{cm}^{3}\right)$, and the range and energy of the ions.

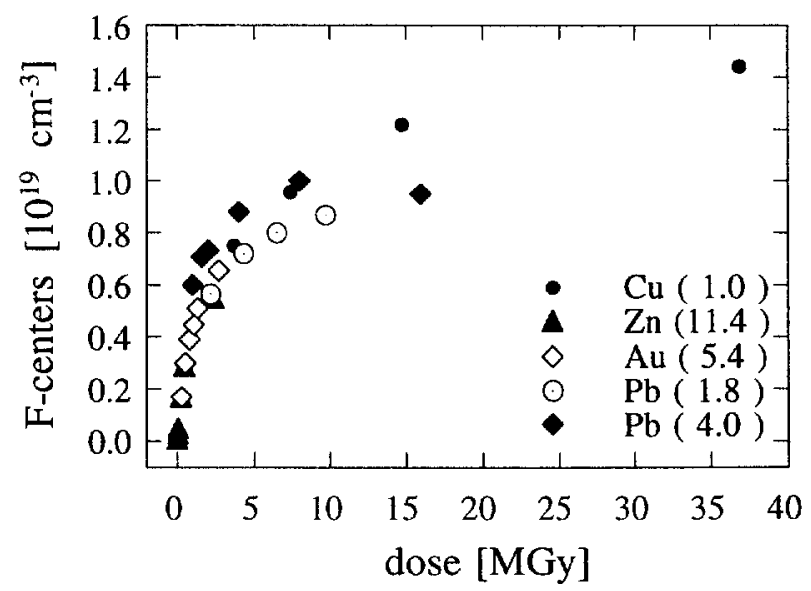

Fig. 4 The concentration of $F$-centers as a function of the absorbed dose for various ions (the energy is given in $\mathrm{MeV} / \mathrm{u}$ )

Although in most cases saturation was not reached, such a presentation (Fig. 4) makes evident that the concentrations of color centers produced by. various ion species is determined by the total amount of the absorbed dose. This is in good agreement with classical damage processes where non-linear effects occur for doses above 0.1 MGy corresponding to a $F$ center concentration of $10^{18} \mathrm{~cm}^{-2}$.

\section{Chemical etching}

In many different materials in particular in insulators (e.g. polymers, mica), ion tracks can be revealed by chemical etching. The process is based on the increased chemical reactivity of the damage along the ion path compared to the unirradiated surrounding matrix. Already in the late fifties, Young [26] had observed that in LiF tracks of uranium fission fragments can be etched in a solution of concentrated hydrofluoric acid (50 vol\%) and glacial acetic acid (50 
vol\%) saturated with ferric fluoride $\left(\mathrm{FeF}_{3}\right)$. To investigate the specific conditions of track etching on a more systematic level, we tested tracks of various ion species in a wide range of energies. At room temperature, the irradiated crystals were immersed in the recommended solution for times between 10 and $30 \mathrm{~min}$. On the irradiated crystal surface, successful etching leads to the evolution of each individual track into a single etch pit as demonstrated on the scanning electron microscopy (SEM) image in Fig. 5.

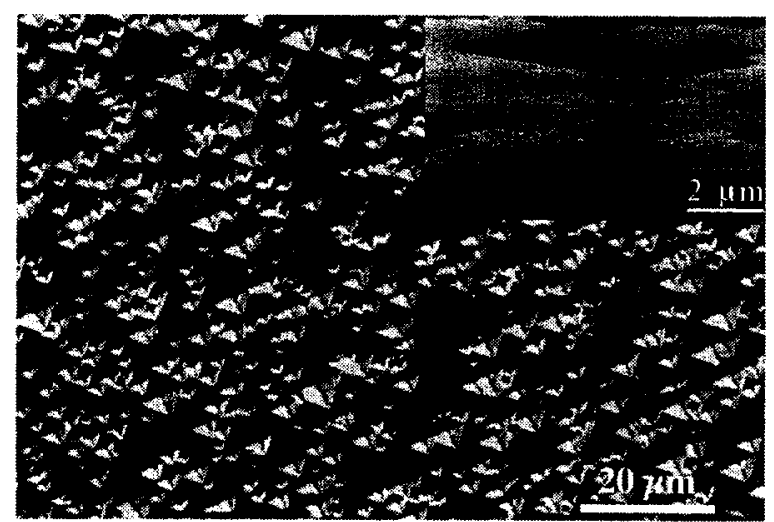

Fig. 5 SEM image of a etched crystal surface irradiated with $10^{7}$ ions $/ \mathrm{cm}^{2}$ (U, $11.4 \mathrm{MeV} / \mathrm{u}$ ) and etched for about $20 \mathrm{~min}$. Each track is revealed by a pyramidal-shaped etch pit characterised by a wide opening angle (inset).

Owing to the cubic structure of the $\mathrm{LiF}$ lattice, the etch pits have a pyramidal shape which is the same geometry known from etched natural dislocations [27]. The cross section of a crystal cleaved perpendicular to the etched surface is shown in the inset of Fig. 5. The wide opening angle of the craters indicates that under the applied conditions, the etching rate along the ion track is only slightly higher than the etching rate of the nonirradiated crystal [28].

Testing the etchability of various ion species, we found that etching is linked to a critical energy loss (e.g., tracks of $11.4 \mathrm{MeV} / \mathrm{u} \mathrm{Zn}$ ions could not be etched). Figure 6 summarises the results obtained for all ion species used in this study. Curves of the electronic energy loss calculated by using the TRIM 89 code [29] are given as a function of the ion energy. Superimposed are the experimental data, where only filled symbols represent successful track etching. The threshold of different ion species showed a slight increase from $11 \mathrm{keV} / \mathrm{nm}$ for Se ions to $15 \mathrm{keV} / \mathrm{nm}$ for Xe ions (dotted line in Fig. 6). This observation can possibly be 
explained by the so-called velocity effect [30] which describes the fact that for a given energy loss, the damage along the ion path is higher if the energy loss value is reached at a lower ion energy. From chemical etching of tracks in an amorphous iron boron alloy, this velocity effect was also assumed to play a role [31].

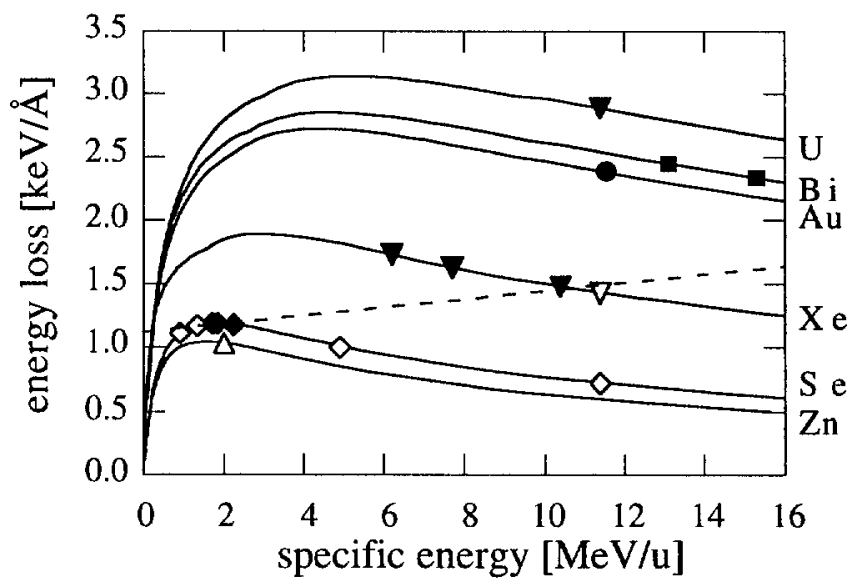

Fig. 6 Calculated curves [29] of the electronic energy loss of various ions as a function of their specific energy. Superimposed are the corresponding experimental data of chemical etching. Tracks were revealed only in those cases (full symbols) where the electronic energy loss surpassed a critical level.

A more impressive manifestation of the threshold effect is obtained when etching the tracks along the ion path. For this, the crystals were cleaved perpendicular to the irradiated sample surface prior to etching. In this geometry, the tracks are revealed as long striated structures as shown in Fig. 7. The large etch pits in the irradiated zone originate from natural dislocations. Their distorted geometry is possibly due to the superposition of dislocation and track etching. 


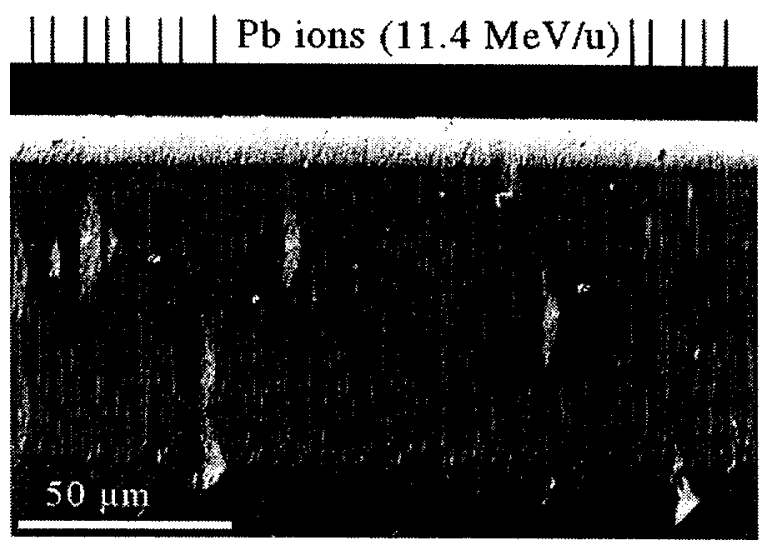

Fig. 7 SEM image of tracks etched along the trajectories of $\mathrm{Pb}$ ions $\left(10^{10} \mathrm{~cm}^{-2}\right)$. The large etch pits originate from natural dislocations. The bright zone at the crystal surface is an artefact due to the cleaving procedure.

While tracks of heavier ion species were etchable along the full length of their path, the situation was different for the lighter $\mathrm{Kr}$ and Se ions. Here, the tracks can only be etched deep in the crystal where the critical energy loss is surpassed. In Figure 8, the etchable zone is visible as $10 \mu \mathrm{m}$ long parallel structures buried in a depth of about $80 \mu \mathrm{m}$ in the bulk of the crystal. It should be emphasised that the transition from the nonetchable to the etchable damage is surprisingly sharp and is only characterised by an increased number of small dislocations (inset of Fig. 8)

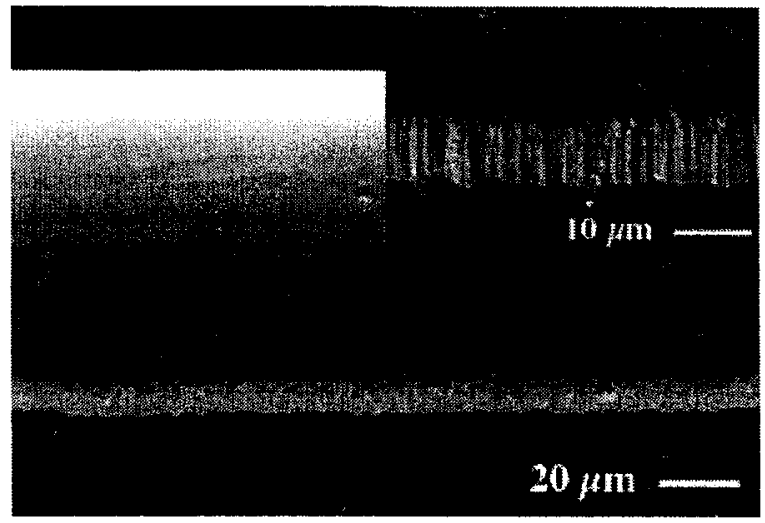

Fig. 8 SEM image of etched trajectories of Se ions. Only in a depth of about 80 $\mu \mathrm{m}$, the damage is etchable and becomes visible as a light zone of parallel structures (inset).

Although the microchemistry of the etching process is not understood, it should be mentioned that the initial stage of etching has recently been studied by atomic force 
microscopy (AFM). First images of ion trajectories etched for only a few seconds do not show a continuous etch line but a sequence of overlapping single etch pits [32].

\section{Small-angle $x$-ray scattering}

In order to complement the results obtained by optical spectroscopy which mainly allowed us to analyse single defects, we also performed small-angle $\mathrm{x}$-ray scattering experiments with crystals irradiated with fluences between $5 \times 10^{9}$ and $2 \times 10^{11}$ ions $/ \mathrm{cm}^{2}$. The measurements were carried out with a set-up in transmission geometry, typically with an angle of $45^{\circ}$ between the ion trajectories and the $\mathrm{x}$-ray beam. We used the $\mathrm{K}_{\alpha}$-Cu radiation of a rotating anode generator ( $49 \mathrm{kV}$ and $200 \mathrm{~mA}$ ) with a pinhole collimation and a position-sensitive area detector of $10 \times 10 \mathrm{~cm}^{2}$ in size.

For the analysis of the intensity distribution, it was assumed that each track can mathematically be described by a cylinder of a Gaussian radial density variation with the zaxis of the cylinder corresponding to the ion trajectory. For such a configuration, the intensity distribution $I\left(k_{r}, k_{z}\right)$ in the reciprocal space is given by

$$
\mathrm{I}\left(\mathrm{k}_{\mathrm{r}}, \mathrm{k}_{\mathrm{z}}\right)=\Delta \rho^{2} \times 4 \pi^{2} \times a^{4} \times \sin ^{2}\left(\mathrm{k}_{\mathrm{z}} \times \mathrm{R} / 2\right) / \mathrm{k}_{\mathrm{z}}^{2} \times \exp \left(-\mathrm{k}_{\mathrm{r}}^{2} \times a^{2} / 2\right),
$$

where $\mathrm{R}$ is the length of the cylindrical track corresponding to the ion range, and $\mathrm{k}_{\mathrm{z}}$ and $\mathrm{k}_{\mathrm{r}}$ is the length of the momentum vector respectively parallel and perpendicular to the ion path. Here, $\mathrm{k}_{\mathrm{r}}$ is obtained from the relation $\mathrm{k}_{\mathrm{r}}=4 \pi / \lambda \sin \Theta$, where $2 \Theta$ denotes the scattering angle and $\lambda(1.54 \AA)$ is the wavelength of the $\mathrm{x}$-ray beam. $\Delta \rho$ is the maximum electron density difference between the damaged material of the track and the virgin crystal, and $a$ defines the Gaussian radius of the track at which $\Delta \rho$ decreases to $1 / \mathrm{e}[33]$. 


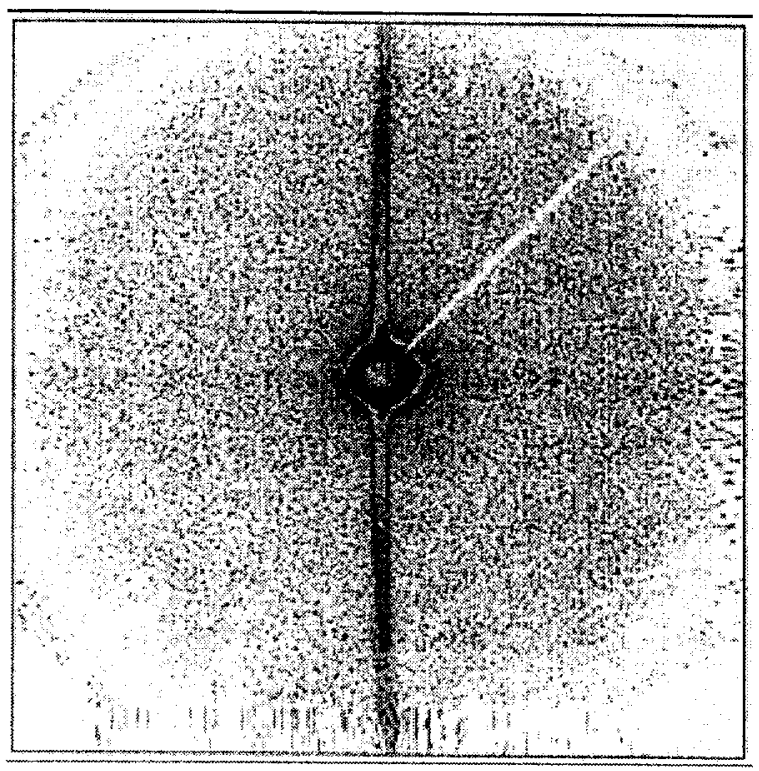

Fig. $9 \quad X$-ray scattering of Xe tracks $\left(10^{10} \mathrm{~cm}^{-2}\right)$ results in a highly anisotropic pattern on the $10 \times 10 \mathrm{~cm}^{2}$ position-sensitive detector.

Figure 9 shows a typical anisotropic scattering pattern where the high contrast along the vertical axis of the detector plane reflects the electron density difference between the damage in the tracks and the surrounding unirradiated crystal. For the lighter $\mathrm{Zn}$ and $\mathrm{Se}$ ions, the anisotropy of the scattering pattern was still visible, but the contrast was too weak to be evaluated. For heavier ions, the scattered intensity was analysed along the vertical cut through the center of the detector plane. Plotting the logarithm of the scattered intensity $I\left(k_{r}\right)$ versus the square of the scattering vector $\mathrm{k}_{\mathrm{r}}$ (Fig. 10), the Gaussian track radius was determined according to Eq. 2. From the excellent fit of the scattering data to a straight line, we conclude that the geometry of the damage along the ion path is well represented by a cylinder. Further evidence for this shape is provided by SAXS experiments where the $\mathrm{x}$-ray beam was oriented perpendicular to the ion tracks $\left(10^{11} \mathrm{Bi}\right.$ ions $\left./ \mathrm{cm}^{2}, 10.9 \mathrm{MeV} / \mathrm{u}\right)$. Under this condition, we obtained the same anisotropic scattering pattern indicating that the damage has a quasicontinuous structure rather than consisting of fragmented damage zones. In this latter case, e.g. spherical defects would lead to an isotropic scattering pattern which was not observed here [34]. 


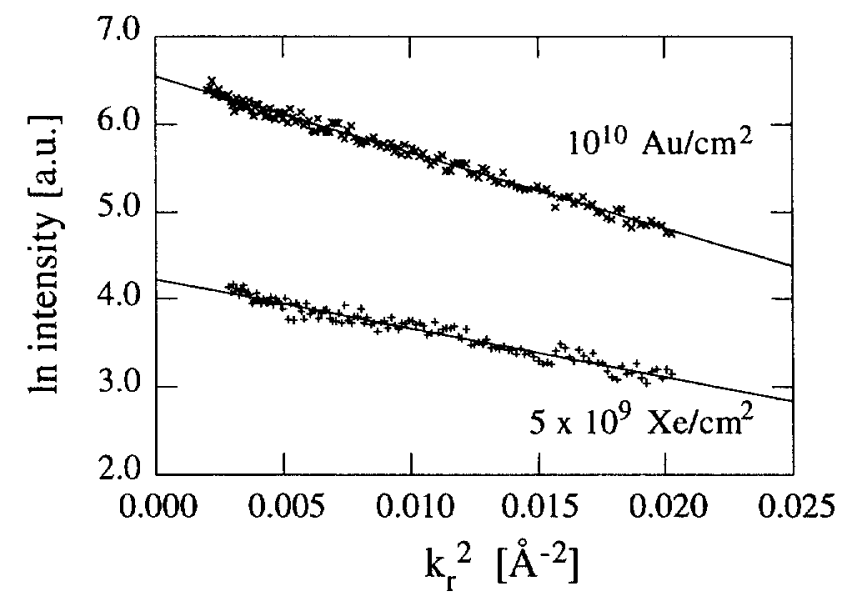

Fig. 10 The scattered $\mathrm{x}$-ray intensity versus the scattering vector $\mathrm{k}_{\mathrm{r}}$ for crystals irradiated with $\mathrm{Xe}$ and $\mathrm{Au}$ ions.

Figure 11 displays the SAXS radius of various ion species increasing as a function of the mean energy loss frorn $1.0 \mathrm{~nm}$ for $\mathrm{Kr}$ ions to $1.8 \mathrm{~nm}$ for $\mathrm{U}$ ions. Each data point represents a mean value of several measurements using different crystals. It should be noted that the scattering intensity results from the integration over the irradiated sample volume and thereby over the contribution of all tracks as scattering objects. Therefore, only a mean SAXS radius can be deduced averaged over the density change along each track and over all contributing tracks. For a given ion, the SAXS radius did not change significantly in the fluences regime between $5 \times 10^{9}$ and $2 \times 10^{11}$ ions $/ \mathrm{cm}^{2}$.

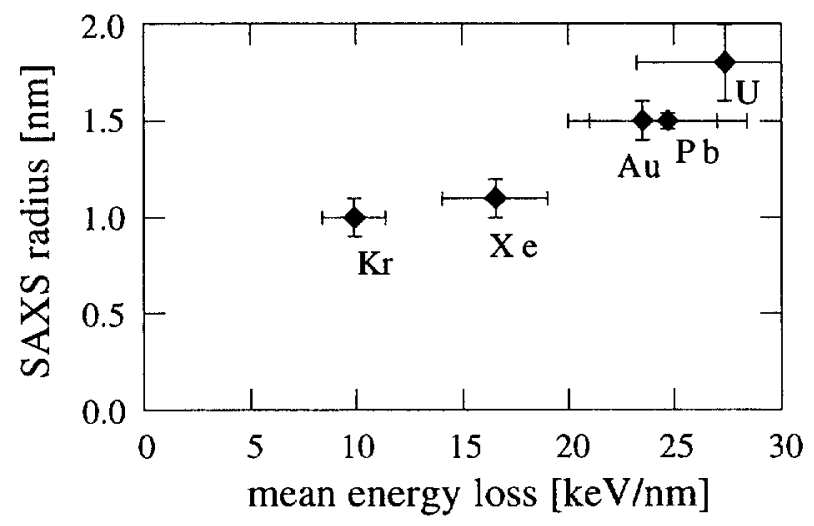

Fig. 11 SAXS radius as a function of the mean energy loss. The initial energy of all ion species was $11.4 \mathrm{MeV} / \mathrm{u}$.

Comparing the SAXS data with those obtained from optical spectroscopy, it is evident that 
the SAXS radii are about one order of magnitude smaller than the region saturated by $F$ centers. Obviously, tracks in LiF have of a structure, i.e., a small core of a few nanometers surrounded by a much larger halo consisting mainly of single $F$ - and hole centers.

\section{Irradiation at low temperatures}

In order to test the influence of temperature on track formation, several crystals were irradiated at different low temperatures between $(15,90,150$, and $300 \mathrm{~K})$ [35]. The selection was based on the experience from low ionising radiation that specific processes mandatory for defect creation are hindered at the following temperatures [1]: At $15 \mathrm{~K}$, the color centers of the primary Frenkel pairs are not mobile. Therefore, the probability of $F$-center-interstitialrecombination is very high restricting the creation of stable defects and defect aggregates. The efficiency to produce defects is expected to increase at $90 \mathrm{~K}$, where the primary $H$-centers are converted into $V_{\mathrm{K}}$-centers (self-trapped holes) and at $150 \mathrm{~K}$, where $V_{\mathrm{K}}$-centers become thermally instable, and the primary hole centers are transformed into more complex hole centers $\left(V_{\mathrm{n}}\right.$-centers $)$.

Surprisingly enough, we observed that for all tested irradiation temperatures track etching was successful. Only the coloration was slightly suppressed as expected from classical irradiation experiments. Also the anisotropic SAXS pattern was observed in all cases. However, the scattering contrast became less pronounced indicating that the local electron density decreased. The SAXS radius of $\mathrm{Pb}$ ions (5.9 MeV/u) slightly increased from $1.2 \mathrm{~nm}$ at $15 \mathrm{~K}$ to about $1.5 \mathrm{~nm}$ for the irradiation at room temperature.

\section{Annealing}

Several irradiated LiF samples were annealed under argon atmosphere for 20 to 30 min up to a temperature of $500^{\circ} \mathrm{C} \mathrm{[34].} \mathrm{In} \mathrm{samples} \mathrm{irradiated} \mathrm{with} \mathrm{a} \mathrm{low} \mathrm{fluences} \mathrm{(e.g.,} \mathrm{Zn}, \mathrm{Au}, \mathrm{Bi}$ of 11.4 MeV/u and $5 \times 10^{9}$ ions $/ \mathrm{cm}^{2}$ ) the concentration of single $F$-centers decreased by a factor of two at $240^{\circ} \mathrm{C}$ and disappeared completely at $340^{\circ} \mathrm{C}$. From an Arrhenius plot, the activation energy for annealing of $F$-centers was determined to be about $0.35 \mathrm{eV}$. This value 
corresponds well with the activation energy for the diffusion of hole centers and suggests that in such a situation, annealing occurs via recombination of hole centers with $F$-centers.

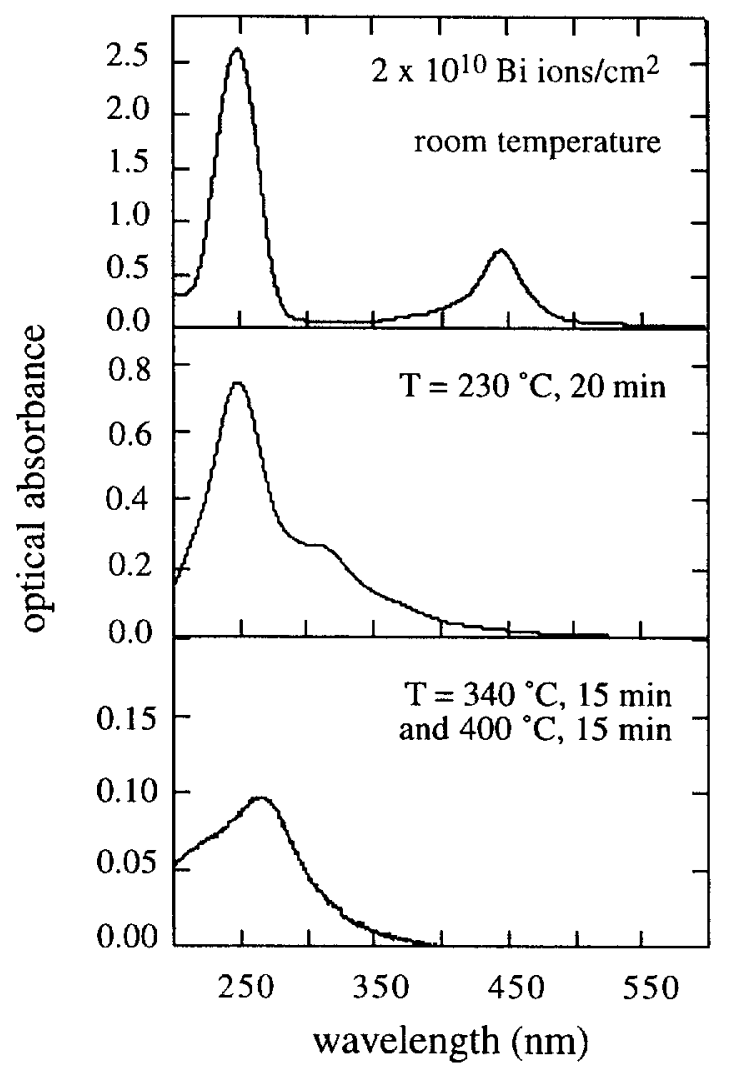

Fig. 12 Absorption spectra of samples irradiated with $2 \times 10^{10} \mathrm{Bi}$ ions $/ \mathrm{cm}^{2}$ $(10.9 \mathrm{MeV} / \mathrm{u})$ and annealed at various temperatures.

The situation was different for higher fluences. Already before the thermal treatment, the absorption spectra of such samples exhibited a complex band structure due to aggregated

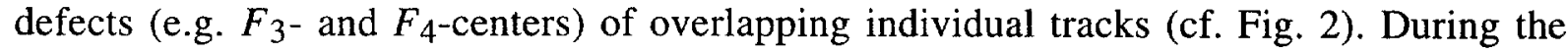
annealing procedure, aggregates of $F$-centers disappeared at a lower temperature than single $F$-centers. Above about $300^{\circ} \mathrm{C}$, the spectrum had only one broad peak at $275 \mathrm{~nm}$ (Fig. 12). Without changing the position of the maximum and the halfwidth of the peak, this band was stable up to $360^{\circ} \mathrm{C}$. At higher temperatures, its intensity started to decrease and finally, the signal disappeared at $450^{\circ} \mathrm{C}$. From this observation, we conclude that the band can be ascribed to rather stable aggregates formed under increased diffusion of single $F$-centers at temperatures above $300^{\circ} \mathrm{C}$. The authors of refs. [5] and [24] also report about a band at 275 
nm obtained after annealing of irradiated LiF crystals (ion implantation, thermal neutrons). Since electron spin resonance experiments gave no colloid signal, it was concluded that this band corresponds to non-paramagnetic aggregates.

In contrast to the color centers, the etchability of tracks was maintained up to much higher temperatures. Below $300^{\circ} \mathrm{C}$, the etch pits exhibited no significant change. Between 300 and $400^{\circ} \mathrm{C}$, the number of etch pits continuously decreased with the disappearance of mainly smaller etch pits. Finally for temperatures above $400^{\circ} \mathrm{C}$, we could not find any etch pits. Results from SAXS experiments of annealed crystals indicate a very similar behaviour. Up to $300^{\circ} \mathrm{C}$, the SAXS radius did not change significantly, between $300^{\circ} \mathrm{C}$ and $400^{\circ} \mathrm{C}$, the anisotropy of the scattered intensity could still be identified but was too weak to be evaluated, and finally above $400^{\circ} \mathrm{C}$, the typical pattern disappeared completely. Obviously, the stability of the damage responsible for the SAXS pattern and the etchability of tracks is maintained up to a higher temperature than $F$-centers.

\section{Swelling}

During various irradiations at higher fluences, we had noticed that the surface of irradiated crystals had bulged outward appreciably. The bending effect was sufficiently great to be detected with the naked eye and would sometimes lead to spontaneous cleaving of the irradiated volume from the unirradiated crystal substrate. Using profilometry, a systematic study was performed in order to quantify this volume expansion by measuring the out-ofplane swelling.

We used $1 \mathrm{~mm}$ thick single crystals with a polished surface (roughness 5-10 nm). During the irradiations, the samples were partially masked in order to analyse the irradiation effects in direct comparison with a virgin area of the same crystal. Due to the fact that the ions were stopped in a thin surface layer of the crystals, the free expansion of the irradiated volume is partially limited by the constraint of the undamaged substrate. The quantitative analysis of the out-of-plane swelling was performed with a profilometer (Dektak 8000) where a high precision stage moves the sample beneath a diamond-tipped stylus over the border line 
between the virgin and the irradiated area of the crystal surface.

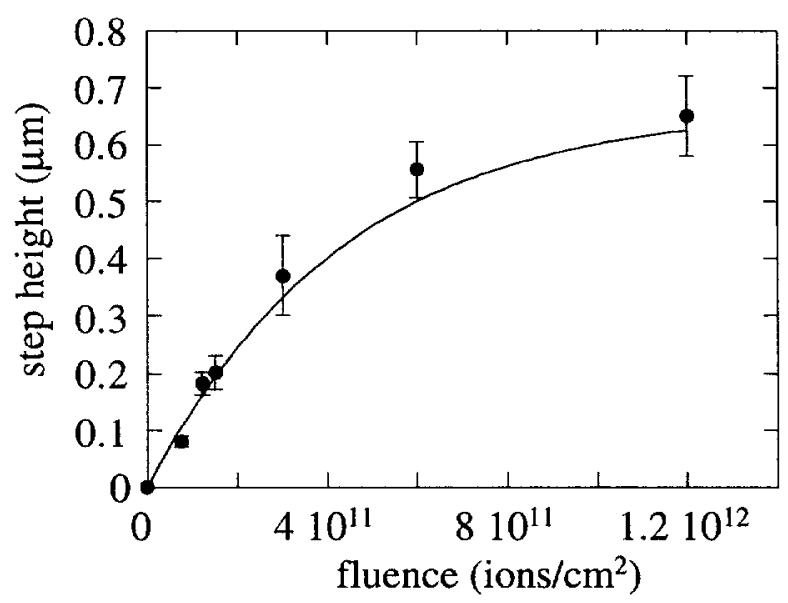

Fig. 13 Step height as a function of the fluence for irradiations with $\mathrm{Pb}$ ions of 4 $\mathrm{MeV} / \mathrm{u}$.

Since a complete analysis of the swelling effects in LiF using various ion species and energies will be published elsewhere, it just should be mentioned that the swelling effect depends on the fluence, the electronic stopping power and the range of the ions in agreement with observations made in various oxides [36-39]. In this report, we want to concentrate on the evolution of the step height as a function of the fluence. Under the irradiations with $\mathrm{Pb}$ ions of $4 \mathrm{MeV} / \mathrm{u}$, the step height initially increases as a linear function, while at high fluences, the swelling approaches saturation (Fig. 13). The solid line corresponds to a fit using the same exponential fluence dependence as described in Eq. 1 from which a radius of about $10 \mathrm{~nm}$ was deduced. The size of this radius lies between the SAXS radius and the halo region and can be regarded as the cylindrical track which contributes to the volume expansion.

\section{Discussion}

The results obtained independently by optical absorption spectroscopy and by SAXS indicate that the damage zone around the trajectory of an energetic ion can be characterised by a small core region with complex defect aggregates surrounded by a much larger halo consisting mainly of simple color centers. It is not possible to study both regions with the 
same experimental technique due to the fact that SAXS is sensitive to the electron density difference between the defect aggregates and the virgin crystal while optical spectroscopy mainly detects single defects. In the fluence regime of individual (that means nonoverlapping) tracks, the concentration of larger defects is too small to be evaluated quantitatively by this technique. If we try to observe the defect clusters in the core region, extremely high fluences are needed. In such a situation, the halo regions would overlap significantly and the signal of aggregated single defects in the halo can not be separated from the defects clusters in the track core.

It should be mentioned that in the single track regime, the surface of various crystals have recently been studied by atomic force microscopy. The impact zones of the ions were imaged as hillocks of several tens of nanometers in diameter and 1-2 $\mathrm{nm}$ in height $[32,40]$. The correlation of the macroscopic swelling and the hillocks as seen by AFM is still an open question and needs further investigations.

The formation of more complex defects in the track halo is related to a critical energy loss of about $10 \mathrm{keV} / \mathrm{nm}$. Results obtained by SAXS and by chemical etching indicate that both techniques test the damage in the core which is formed even at an irradiation temperature as low as $15 \mathrm{~K}$. This effect is surprising because at such a low temperatures, the primary Frenkel defects in LiF crystals are not mobile and aggregation processes are strongly suppressed.

The nature of the etchable damage has never been identified. However, based on the following arguments, we assume that the etchability of tracks and also the damage which causes the SAXS signal is strongly related to the formation of larger defect aggregates: (i) it was demonstrated that in LiF crystals irradiated with thermal neutrons, defect clusters can be etched whereas evidence for the etchability of single defects or small aggregates (e.g. $F_{2}$ - or $F_{3}$-centers) has never been found [6], (ii) the etchability and the SAXS pattern of tracks is maintained up to much higher temperatures than the survival of single defects.

If we assume that under ion irradiation similar defect processes occur as known from conventional radiation, it can be expected that above a critical dose, defect clusters such as 
colloids, fluorine clusters, and vacancy clusters are formed with single defects $(F$-centers and hole centers) as precursors. As a consequence of the coagulation of $F$-centers to larger $\mathbf{n} F$ aggregates, the ionic lattice collapses and metallic colloids of reduced density are formed [810]. If the same process takes place along the path of heavy ions and if a continuous trail of metallic colloids is formed, this should lead to a change of the electrical properties. We measured the dc surface conductivity perpendicular to the tracks and the ac conductivity from $10 \mathrm{~Hz}$ to $1 \mathrm{MHz}$ along the tracks at room temperature. In both cases, no significant change of the conductivity was observed not even for samples irradiated at the highest fluence of $1.5 \mathrm{x}$ $10^{13} \mathrm{~Pb}$ ions $/ \mathrm{cm}^{2}$ (4 MeV/u). Measurements of the dielectric constant $\varepsilon$ at temperatures as low as $77 \mathrm{~K}$ gave also no indication of any modification compared to the pristine crystal. Furthermore, crystals irradiated with $5 \times 10^{11} \mathrm{Au}$ ions $/ \mathrm{cm}^{2}(11.4 \mathrm{MeV} / \mathrm{u})$ were tested by electron spin resonance (EPR) experiments. Also here, no significant signal was found which could be ascribed to metallic colloids. Using the information we obtained about the size of the track core and the number of $F$-centers in a single track (about $10^{6}$ for $U$ ions), we can estimate that the number of $\mathrm{Li}$ atoms available for the colloid formation reaches only a few percent [34]. Such a small Li concentration does not allow the formation of a continuous trail of metallic Li colloids and possibly explain the results of the EPR and conductivity tests.

\section{Conclusions}

Experimental observations using various techniques such as optical absorption, chemical etching, and small-angle $\mathrm{x}$-ray spectroscopy give evidence that tracks in lithium fluoride exhibit a complex structure and defect morphology (Fig. 14). Single defects such as $F$-centers are produced in a large halo of several tens of nanometers around the ion trajectory. The damage processes in the halo are similar to those under conventional radiation. If the ions surpass a critical energy loss of about $10 \mathrm{keV} / \mathrm{nm}$, a new phenomenon occurs namely the creation of complex defect clusters in a narrow zone of about 2-4 $\mathrm{nm}$ in diameter. Evidence for such defect aggregates is given by a characteristic anisotropic SAXS pattern and by the etchability of ion tracks. In the range between 15 and $300 \mathrm{~K}$, this effect seems to be almost 
independant from the irradiation temperature. Although the nature of this specific damage has not been uncovered so far, it is assumed that more complex defect aggregates such as small $\mathrm{Li}$ colloids, and fluorine and vacancy clusters are responsible for the observed effects. Furthermore, profilometer measurements reveal substantial volume expansion under ion irradiation. The effect can be assigned to a track radius of about $10 \mathrm{~nm}$ which corresponds to an intermediate zone between the track core and halo. Finally it should be emphasised that this complex track structure is due to the radiation sensitivity of lithium fluoride in combination with the high stability of single defects and directly reflects the extremely high gradient of the spatial dose distribution around the ion path.

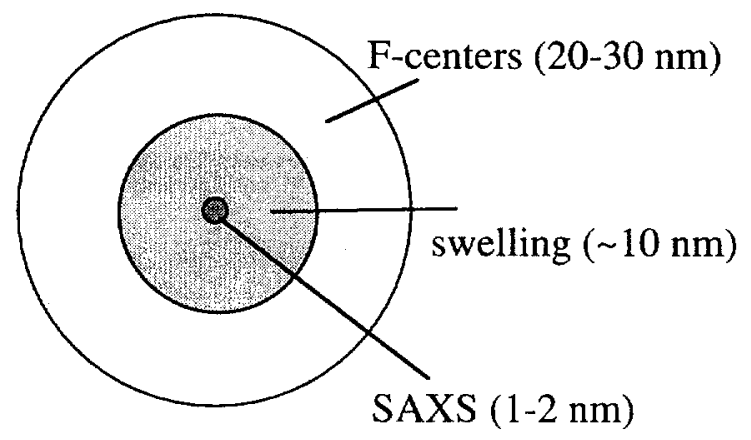

Fig. 14 The structure of tracks in LiF is described by (1) a large halo mainly consisting of single color centers, (2) a medium sized zone contributing to swelling, and (3) a small core region responsible for etching and SAXS.

Acknowledgement

We would like to thank F. Beuneu for performing the electron-spin resonance experiments.

\section{References}

[1] N. Itoh and K. Tanimura, J. Phys. Chem. Solids 51 (1990) 717.

[2] N. Itoh, Nucl. Instr. Meth. B 122 (1997) 405.

[3] Ch.B. Lushchik, in Physics of Radiation Effects in Crystals, Ed. R. A. Johnson and A. N. Orlov (Elsevier, Amsterdam,1986) pp. 473 -525.

[4] C.R.A. Catlow, K.M. Diller, L.W. Hobbs, Philos. Mag. A42 (1980) 123.

[5] A.T. Davidson, J.D. Comins, A.M. J. Raphuthi, A.G. Kozakiewicz, E.J. Sendezera, and T.E. Derry, J. Phys.: Condens. Matter 7 (1995) 3211.

[6] J.J. Gilman and W.G. Johnston, J. Appl. Phys. 29 (1958) 877. 
[7] P. Durand, Y. Farge, and M. Lambert. J. Phys. Chem. Solids 30 (1969) 1353.

[8] R. Kaplan and P.J. Bray, Phys. Rev. 129 (1963) 1919.

[9] C.D. Knutson, H.O. Hoopers, and P.J. Bray, J. Phys. Chem. Solids 27 (1966) 147.

[10] A.E. Hughes and S.C. Jain, Adv. Physics 28 (1997) 717.

[11] W.L. McLaughlin, Rad. Protection Dosimetry 60 (1996) 197.

[12] W. Demtröder in: Laser spectroscopy, (Springer, Heidelberg, 1981) 334.

[13] A.M. Stoneham, Nucl. Instr. Meth. B48 (1990) 389.

[14] N. Betz, A. Le Moël, E. Balanzat, J.M. Ramillon, J. Lamotte, J.P. Gallas, and G. Jaskierowitcz, J. Polym. Sci. Polym. Phys. B 32 (1994) 1493.

[15] T. Steckenreiter, E. Balanzat, H. Fuess, C.Trautmann, Nucl. Instr. and Meth. B 131 (1997) 159.

[16] M. Toulemonde, S. Bouffard, and F. Studer, Nucl. Instr. and Meth. B 91 (1994) 108.

[17] A. Meftah, F. Brisard, J.M. Costantini, E. Dooryhee, M. Hage-Ali, M. Hervieu, J.P. Stoquert, F. Studer, and M. Toulemonde, Phys. Rev. B 49 (1994) 12457.

[18] A. Perez, E. Balanzat, and J. Dural, Phys. Rev. B 41 (1990) 3943.

[19] E. Balanzat, S. Bouffard, A. Cassimi, E. Dooryhee, L. Protin, J.P. Grandin, J.L. Doualan, and J. Margerie, Nucl. Instr. Meth. B 91 (1994) 134.

[20] P. Thévenard, G. Guiraud, C.H.S. Dupuy, and B. Delaunay, Radiation Effects 32 (1977) 83.

[21] A. Perez, M. Döbeli, and H.A. Synal, Nucl. Instr. Meth. B 116 (1996)13.

[22] A. Smakula, Z. Physik 59 (1930) 603.

[23] J.H. Crawford, Adv. Phys. 17 (1968) 93.

[24] N.G. Politov, L.F. Vorozheikina, Soviet Physics Solid State 12 (1970) 277.

[25] N. Seifert, H. Ye, N. Tolk, W. Husinsky, and G. Betz, Nucl. Instr. Meth. B 84 (1994) 77.

[26] D.A. Young, Nature 182 (1958) 375.

[27] J.J. Gilman and W.G. Johnston, J. Appl. Phys. 29 (1958) 747.

[28] C. Trautmann, K. Schwartz, O. Geiß, J. Appl. Phys. 83 (1998) 3560.

[29] J.F. Ziegler, P. Biersack, and U. Littmark in: The Stopping and Ranges of Ions in Matter, ed. J.F. Ziegler (Pergamon, New York, 1985).

[30] A. Meftah, F. Brisard, J.M. Costantini, M. Hage-Ali, J.P. Stoquert, F. Studer, and M.Toulemonde, Phys. Rev. B 48 (1993) 920.

[31] C. Trautmann, M. Toulemonde, Ch. Dufour, and E. Paumier, Nucl. Instr. Meth. B 108 (1996) 94.

[32] A. Müller, R. Neumann, K. Schwartz, T. Steckenreiter, and C. Trautmann, Appl. Physics A 29 (1998) 1147.

[33] D. Albrecht, P. Armbruster, and R. Spohr, M. Roth, K. Schaupert, H. Stuhrmann, Appl. Phys. A 37 (1985) 37. 
[34] K. Schwartz, C. Trautmann, T. Steckenreiter, O. Geiß, M. Krämer, accepted for publication in Phys. Rev. B

[35] K. Schwartz, G. Wirth, and C. Trautmann, Phys, Rev. B 56 (1997) 10711.

[36] B. Canut, R. Brenier, A. Meftah, P. Moretti, S. Ould Salem, S.M.M. Ramos, P. Thévenard, M. Toulemonde, Nucl. Instr. Meth. Phys. Res. B 91 (1994) 312

[37] R.Brenier, B. Canut, S.M.M.Ramos, P. Thévenard, Nucl. Instr. Meth. Phys. Res. B 90 (1994) 339.

[38] C. Trautmann, J.M. Costantini, A. Meftah, K. Schwartz, J.P. Stoquert, M. Toulemonde, accepted for publication in Atomistic Mechanisms in Beam Synthesis \& Irradiation of Materials (1998).

[39] M. Toulemonde, A. Meftah, J.M. Costantini, K. Schwartz, C. Trautmann, this conference.

[40] A. Müller, R. Neumann, K. Schwartz, C. Trautmann, this conference. 